\title{
Characteristics of hand-to-environment contact during indoor activities in daily life among Korean adults using a video-based observation method
}

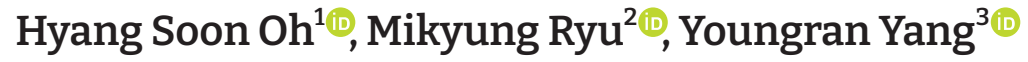 \\ ${ }^{1}$ Department of Nursing, College of Life Science and Natural Resources, Sunchon National University, Suncheon, Korea \\ ${ }^{2}$ Department of Nursing, College of Nursing and Public Health, Daegu University, Daegu, Korea \\ ${ }^{3}$ College of Nursing, Research Institute of Nursing Science, Jeonbuk National University, Jeonju, Korea
}

Received: January 9, 2021

Revised: May 6, 2021

Accepted: May 13, 2021

Corresponding author:

Mikyung Ryu

Department of Nursing, College of Nursing and Public Health, Daegu University, 33 Seongdang-ro 50-gil, Nam-gu, Daegu 42400, Korea E-mail: ryumk@daegu.ac.kr

\section{ABSTRACT}

Objectives: The aim of this study was to describe the characteristics of hand-to-environmental contact (HEC) and to identify the factors influencing HEC behavior in Korean adults' indoor daily life.

Methods: Thirty participants were enrolled from January 14 to February 12, 2018 after providing informed consent for being videotaped. Data were collected by recording their indoor daily lives for 2 hours, resulting in 4,732 HEC cases. To ensure the accuracy and reliability of the HEC readings, 3 training sessions were conducted for the videotape readers. Rereading and verifying randomly selected data ensured the validity of intra- and inter-reader readings.

Results: The most frequent contact items were phones, papers, computer accessories, and furniture surfaces. The contact density (frequency-duration/min) was highest for category II (items occasionally shared by others, 56.8), followed in descending order by category I (items for individual use, 35.9), and category III (public use items, 3.4). Significant differences in contact density were found according to participants' demographic characteristics.

Conclusion: As mobile phones were the most frequent contact item, regular and strict mobile phone cleansing or disinfection strategies are needed, in addition to preventative measures taken for category II and III items. Avoiding sharing personal items with others, refraining from unnecessary HEC, and maintaining strict hand hygiene are recommended.

Keywords: Activities of daily living; Contact tracing; Disease transmission; Environment; Hand hygiene

\section{Introduction}

Hand contact is the most frequent mode of infection transmission [1,2]. Environmental contamination is an increasingly serious issue in terms of infectious pathogen transmission 
through the contact mode of transmission [3,4]. Environmental surfaces can be easily contaminated with various pathogens and can act like a "reservoir" for these pathogens, facilitating their transmission by hand contact with contaminated environmental surfaces [5,6]. Indoor human environments, including homes, offices, schools, workplaces, transport systems, and other settings, can often be sources of potentially unsafe microorganisms [5-8]. This issue is also a concern for respiratory viruses, since adults who are sick with colds commonly contaminate environmental surfaces with the causative rhinovirus. If aerosol droplets settle on these surfaces, viruses can remain transmissible for hours or days $[5,9]$.

A previous study that investigated indoor environmental contamination with a rhinovirus reported that $35 \%$ of the 150 environmental sites in the rooms were contaminated [10]. Common virus-positive sites were frequent hand-contact sites, such as door handles, pens, light switches, TV remote controls, faucets, and telephones. Moreover, the rhinovirus was transferred from surfaces to fingertips in 18 out of 30 trials (60\%) 1 hour after contamination and in 10 out of 30 trials (33\%) 18 hours (overnight) after contamination. In 2015, during the Middle East respiratory syndrome (MERS) outbreak in the Republic of Korea (hereafter Korea), environmental contamination was suggested as a potential mode of MERS transmission [11].

In terms of person-to-person spread, a study reported that household transmission involving at least 14 people could occur through horizontal spread, one person after the other, by touching the same contaminated door handle [12]. Successive transmission from one person to another was shown to spread up to the sixth contact person under everyday living conditions in an apartment shared by 4 students [12].

The above findings show that not only aerosol droplets, but also frequent hand contact with environmental surfaces can be a mode of easy contamination, increasing the risk of infection. To decrease the risk of the transmission of infection through hand-to-environment contact (HEC), reducing the contact rate is somewhat more effective than increasing ventilation $[6,13,14]$.

It is therefore important to assess the frequency and characteristics of HEC to identify the degree of exposure and infection risk through this route. In recent years, there has been increasing research on environmental contamination with microorganisms and hand contact with the environment [15-17]. However, few studies have been conducted in Korea about HEC in daily life. Infectious disease outbreaks in Korea, such as MERS in 2015, may be expected to occur more frequently and more severely in the future, because
Korea is a hot spot for expanding international travel and business. Thus, it is necessary to implement infectious disease outbreak preparedness and control measures. The purpose of this study was, therefore, to describe the characteristics of HEC in Korean adults' indoor daily life activities to identify HEC-related behavioral factors as a pilot study.

\section{Materials and Methods}

\section{Participants and Data Collection}

This study used a video-based observation method to quantify HEC behaviors in the daily activities of Korean adults. Thirty participants were recruited and video recordings of their daily indoor activities were collected as research data from January 14 to February 12, 2018. The purpose of this study and the method of data collection were explained to the participants and their consent to being videotaped was obtained [18]. The participants were assured of the confidentiality of data to protect their privacy after observing their behaviors through the videotapes. To minimize the participants' behavioral changes as a result of the video observations, they were not informed that the study would involve observation of them touching specific environmental items. We selected videotaping locations for observing indoor activities in daily living environments that were restricted from external access, such as the lecture rooms of the undergraduate students participating in the study and the participants' workplaces and places of worship. Videotaped data were collected for 2 hours during the day when the participants were the most active in terms of performing their daily routine tasks, such as working on the computer, reading, writing, or praying in church.

Trained video readers observed the videos and compiled the observed environmental contact items and the duration of contact in a standardized Microsoft Excel format. The contact duration in terms of time was entered as the start minute, the start seconds to end minute, and end seconds (with reference to time as shown at the bottom of the video player) to avoid missing data. If the time of any reading was missing, it was rechecked via video playback to confirm the actual data.

\section{Classification of Environmental Items for Risk of Contact Transmission}

The environmental items were classified into 3 categories according to the degree to which they are shared with others: category I (items used by the individual); category II (items mainly used by the individual but occasionally used by other people); and category III (public use items). 


\section{Validity and Reliability of Video Data Reading}

Two video readers who attended 3 training sessions crosschecked the videotape readings to confirm their accuracy and reliability. To check for intrapersonal reading errors, $4 \%$ of the random data entered by each video reader was reread and confirmed for contact items and contact duration to ensure a minimum $90 \%$ conformance in the data [18]. The interpersonal errors in reading were reassessed for a minimum 90\% agreement between the 2 readers by randomly selecting and assessing approximately $10 \%$ of the data originally entered by each reader. If the results of the readings differed, the researchers reviewed the case with the readers until consensus was reached.

\section{Contact Density}

We developed the measure of "contact density" to quantify contact intensity over a given time. This measure is obtained by multiplying contact frequency (number/person) by contact duration (sum of contact duration and min/person) and then dividing it by the given observation time in minutes (frequency-duration/min/person). High contact density means an increased risk of potentially transmitting a microorganism.

\section{Data Analysis}

Descriptive statistics were summarized as frequencies, means, standard deviations, and quantiles. If the normality test of the residuals showed the data as normally distributed, means, standard deviations, and the t-test or ANOVA were used. For non-normal data, the median value, first and third quartiles, and the non-parametric Wilcoxon test or the Kruskal-Wallis test were used. A statistical significance level of less than 0.05 was used. Data analysis was conducted using the R 3.3.3 program ver. 3.15 for Windows [19] and the figures were obtained using the package's g plots [20].

\section{Ethics Statement}

We obtained institutional review board (IRB) approval from the human subjects committee of the Sunchon National University (IRB No: 1040173-201712-HR-033-02). Informed written consent was obtained from all individual participants before and after video recording. All video analyses were secured to protect the identities of the participants after video reading.

\section{Results}

\section{Characteristics}

The participants' demographic characteristics are shown in Table 1. Table 2 presents the study characteristics, including the survey location, situation, and observation time.

\section{Observed HEC Items by Subgroup of Environmental Items}

A total of 30 participants were observed for 2 hours each, and 6,078 instances of contact with environmental items were observed during the total of 60-person hours. Finally, 4,732 cases were analyzed, excluding 1,346 cases involving unidentifiable items or those lacking information for the classification of environmental items. Among category I items, mobile phones were found to have the most frequent contact; in category II, papers and computer accessories were the most frequently contacted; and among category III items, furniture surfaces were found to be the most frequently contacted (Table 3).

\section{Contact Frequency and Contact Duration of Specific Items}

Of the environmental contact items, phone contact-including mobile phones-(38.1/person) was most frequently observed,

Table 1. Demographic characteristics of participants $(N=30)$

\begin{tabular}{|c|c|}
\hline Variable & $N(\%)$ \\
\hline Age $(y)$, mean $\pm S D$ & $41.0 \pm 18.5$ \\
\hline $20-29$ & $13(43.3)$ \\
\hline $30-59$ & $10(33.3)$ \\
\hline$\geq 60$ & $7(23.3)$ \\
\hline \multicolumn{2}{|l|}{ Sex } \\
\hline Male & $11(36.7)$ \\
\hline Female & $19(63.3)$ \\
\hline \multicolumn{2}{|l|}{ Marital status } \\
\hline Single & $14(46.7)$ \\
\hline Married & $16(53.3)$ \\
\hline \multicolumn{2}{|l|}{ Education $(N=29)$} \\
\hline$\leq$ High school & $15(51.7)$ \\
\hline$\geq$ College & $14(48.3)$ \\
\hline \multicolumn{2}{|l|}{ Occupation } \\
\hline None & $5(16.7)$ \\
\hline Student & $11(36.7)$ \\
\hline Officer & $5(16.7)$ \\
\hline Others & $9(30.0)$ \\
\hline \multicolumn{2}{|l|}{ Household size (person) } \\
\hline$<3$ & $15(50.0)$ \\
\hline$\geq 3$ & $15(50.0)$ \\
\hline \multicolumn{2}{|l|}{ Household monthly income $(\# 10,000)(N=28)$} \\
\hline$<300$ & $7(25.0)$ \\
\hline 300 to $<500$ & $8(28.6)$ \\
\hline 500 to $<1,000$ & $11(39.3)$ \\
\hline$\geq 1,000$ & $2(7.1)$ \\
\hline \multicolumn{2}{|l|}{ Residence } \\
\hline Metropolis (Seoul) & $18(60.0)$ \\
\hline Urban or suburban (Gyeonggi-do, Jeollanam-do) & $12(40.0)$ \\
\hline
\end{tabular}

SD, standard deviation; \#, Korean won. 
Table 2. Characteristics of the survey location, situation, and observation time of this study

\begin{tabular}{lcllcc}
\hline $\begin{array}{c}\text { Survey } \\
\text { round }\end{array}$ & Participant & \multicolumn{1}{c}{ Survey location } & Daily life (situation) & $\begin{array}{c}\text { Hour of } \\
\text { survey }\end{array}$ & $\begin{array}{c}\text { Total } \\
\text { observation } \\
\text { time (h) }\end{array}$ \\
\hline First & 10 & Chapel in church (Gyeonggi-do) & Congregation worship & 2 & 20 \\
Second & 10 & Classroom in university (Seoul) & Extracurricular activities & 2 & 20 \\
Third & 8 & Administrative office in university (Jeollanam-do) & Administrative work & 2 & 16 \\
Fourth & 2 & Lecture room in university (Jeollanam-do) & Consultation and education & 2 & 4 \\
Total & 30 & & & 8 & 60
\end{tabular}

a) Participant $\times$ hours of survey (person-hour).

Table 3. Top 5 observed items of hand-to-environmental contact (2-h observation time, $n=4,732$ )

\begin{tabular}{|c|c|c|c|c|c|c|}
\hline \multirow{2}{*}{ Rank } & \multicolumn{2}{|c|}{ Category ${ }^{a)}$ । } & \multicolumn{2}{|l|}{ Category II } & \multicolumn{2}{|c|}{ Category III } \\
\hline & Item & $n(\%)$ & Item & $n(\%)$ & Item & $n(\%)$ \\
\hline 1 & Phone $^{\text {b) }}$ & $1,143(24.2)$ & Paper & $603(12.7)$ & Furniture surface & $696(14.7)$ \\
\hline 3 & Hot pack & $94(2.0)$ & Tableware and cooking utensil & $357(7.5)$ & Door handle & $1(0.0)$ \\
\hline 4 & Glass & $82(1.7)$ & Book & $357(7.5)$ & - & - \\
\hline 5 & Tissue & $48(1.0)$ & Document file & $200(4.2)$ & - & - \\
\hline $\begin{array}{l}\text { Total } \\
\text { observation }\end{array}$ & - & $2,113(44.7)$ & - & $1,917(40.5)$ & - & $702(14.8)$ \\
\hline $\begin{array}{l}\text { Contact } \\
\text { frequency }\end{array}$ & - & $70.4 \pm 34.6$ & - & $91.3 \pm 57.0$ & - & $23.4 \pm 22.7$ \\
\hline
\end{tabular}

SD, standard deviation.

${ }^{a)}$ Category I, items used by the individual; category II, items mainly used by the individual but occasionally used by other people; category III, public use

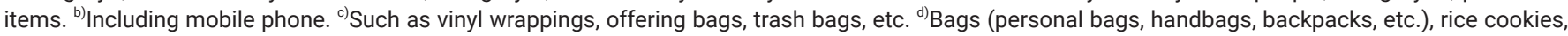
cups, wallets, necklaces, candy, pencils, handkerchiefs, keys, pencil cases, straws, and credit cards. ${ }^{\mathrm{e}}$ Sheet music, snacks, memo papers, hand creams, calendars, cushions, and so forth. ${ }^{f} \mathrm{n} /$ person; mean \pm SD.

followed by contact with furniture and fixture surfaces (23.2/ person) and computer accessories (12.5/person). Other contact items were daily necessities and office supplies (Table 4). The average contact duration per person per contact was 25.8 seconds for phones, including mobile phones, 20.9 seconds for furniture, and 14.0 seconds for tableware and cooking utensils (Table 4).

\section{Descriptive Statistics of HEC by Subgroup of Environmental Items}

The average contact duration of HEC was highest for category I items, and the average contact frequency of HEC was highest for category II items. The average duration of each contact per person was found to be 47.8 seconds for category I, 37.2 seconds for category II, and 20.9 seconds for category III. The average contact time per person was 52.5 minutes for category I, 55.4 minutes for category II, and 7.9 minutes for category III. The average contact density (frequencyduration/ $\mathrm{min}$ ) was 35.9 for category I, 56.8 for category II, and 3.4 for category III (Table 5). As can be seen in Table 3 , which presents the distribution of frequency of contact, contact duration, and contact density, some extremely high values (i.e., outliers) were found in the top quartile.

\section{Histogram for the Distribution of Contact Density by Subgroup Items}

Figure 1 shows that the distribution of contact density was skewed to the left or negatively skewed, especially for category II and III items. This means that there were many occurrences in the lower range of contact density (left side) and few in the upper range (right side) (Figure 1).

\section{Differences in Frequency, Duration, and Density of HEC by Participants' Characteristics}

Table 6 shows that the average density of contact with category I items was significantly higher among unmarried individuals and individuals with a high household income than among married people and in those with a low income, respectively. The results also demonstrate that the average contact density for category II items (papers, computer accessories, utensils, etc.) was different in terms of sex (higher in women). For category III items (furniture surfaces, 
Table 4. Contact frequency and contact duration of the specific items $(N=30)$

\begin{tabular}{lll}
\hline Specific item & Mean \pm SD & Median (range) \\
\hline No. of contacts per person for $2 \mathrm{~h}$ & & $28(0-141)$ \\
Phone (including mobile phone) & $38.1 \pm 39.0$ & $0(0-150)$ \\
Computer accessory & $12.5 \pm 38.7$ & $1(0-56)$ \\
Tableware and cooking utensil & $11.9 \pm 15.5$ & $16(2-103)$ \\
Furniture surface & $23.2 \pm 22.6$ & $27(0-60)$ \\
Contact duration per contact (s/person) & & $0(0-55)$ \\
Phone (including mobile phone) & $25.8 \pm 17.5$ & $4(0-73)$ \\
Computer accessory & $5.2 \pm 15.8$ & $13(3-82)$ \\
Tableware and cooking utensil & $14.0 \pm 19.9$ & $20.9 \pm 20.1$ \\
Furniture surface & &
\end{tabular}

SD, standard deviation.

Table 5. Descriptive statistics of hand-to-environmental contact by subgroup items for risk of contact transmission (2-h observation time, $n=4,532, N=30$ )

\begin{tabular}{|c|c|c|c|c|c|c|c|}
\hline \multirow{2}{*}{ Classification item } & \multirow{2}{*}{$n$} & \multirow{2}{*}{ Mean \pm SD } & \multicolumn{5}{|c|}{ Quantile distribution } \\
\hline & & & 0.1 & 0.25 & 0.5 & 0.75 & 0.9 \\
\hline \multicolumn{8}{|c|}{ Contact duration (s/contact/person) } \\
\hline Category ${ }^{a)} \mid$ & 2,113 & $47.8 \pm 29.5$ & 22.9 & 26.2 & 41.6 & 59.3 & 79.5 \\
\hline Category II & 1,917 & $37.2 \pm 19.1$ & 15.6 & 23.9 & 35.9 & 43.8 & 52.8 \\
\hline Category III & 702 & $20.9 \pm 20.1$ & 7.1 & 7.9 & 13.0 & 22.7 & 52.3 \\
\hline \multicolumn{8}{|c|}{ Contact frequency ( $n /$ person) } \\
\hline Category I & 2,113 & $70.4 \pm 34.6$ & 32.2 & 42.0 & 72.0 & 85.5 & 119.5 \\
\hline Category II & 1,917 & $91.3 \pm 57.0$ & 46.0 & 51.0 & 81.0 & 104.0 & 184.0 \\
\hline Category III & 702 & $23.4 \pm 22.7$ & 3.0 & 8.3 & 15.5 & 31.8 & 48.1 \\
\hline \multicolumn{8}{|c|}{ Sum of contact duration (min/person) } \\
\hline Category I & 2,113 & $52.5 \pm 29.9$ & 15.3 & 33.3 & 50.3 & 76.1 & 93.5 \\
\hline Category II & 1,917 & $55.4 \pm 38.8$ & 27.0 & 32.0 & 47.1 & 59.6 & 120.7 \\
\hline Category III & 702 & $7.9 \pm 11.9$ & 0.7 & 2.2 & 3.7 & 8.6 & 16.0 \\
\hline \multicolumn{8}{|l|}{ Contact density ${ }^{\mathrm{b})}$} \\
\hline Category I & 2,113 & $35.9 \pm 29.4$ & 5.1 & 9.6 & 28.5 & 54.5 & 79.4 \\
\hline Category II & 1,917 & $56.8 \pm 76.7$ & 11.0 & 18.8 & 31.0 & 43.1 & 222.2 \\
\hline Category III & 702 & $3.4 \pm 9.7$ & 0.03 & 0.13 & 0.6 & 1.5 & 5.6 \\
\hline
\end{tabular}

SD, standard deviation.

${ }^{\text {a) }}$ Category I, items used by the individual (mobile phones, personal pens, glasses, etc.); category II, items mainly used by the individual but occasionally used by other people (papers, computer accessories, and utensils, etc.); category III, public use items (furniture surfaces, door handles, etc.); ${ }^{\text {b) }}$ Contact density $=$ contact frequency $\times$ contact duration / observation time (min) (frequency-duration/min/person).

door handles, etc.), there were differences in contact density according to age (higher contact density in older individuals) and education (higher in those with low education levels).

\section{Discussion}

In everyday life, infections constantly spread via HEC [10]. The aim of this study was to understand the characteristics of HEC and to identify factors related to HEC behaviors among Korean adults. According to the Korean Statistical Information Service (KOSIS) [21], adults in Korea spend, on average, 7 hours and 39 minutes daily on compulsory activities such as work, study, and housework, while they spend about 4 hours and 47 minutes on leisure activities, such as dating, participation in group activities or hobbies, and media use. Our results show that there are many opportunities for HEC in everyday life. This study, with its 2-hour recording duration, can be divided into $26.1 \%$ work-related activities and $41.8 \%$ leisure-related activities. However, exact proportions could not be calculated, because the KOSIS report does not distinguish between time spent on indoor activities or on outdoor activities. 

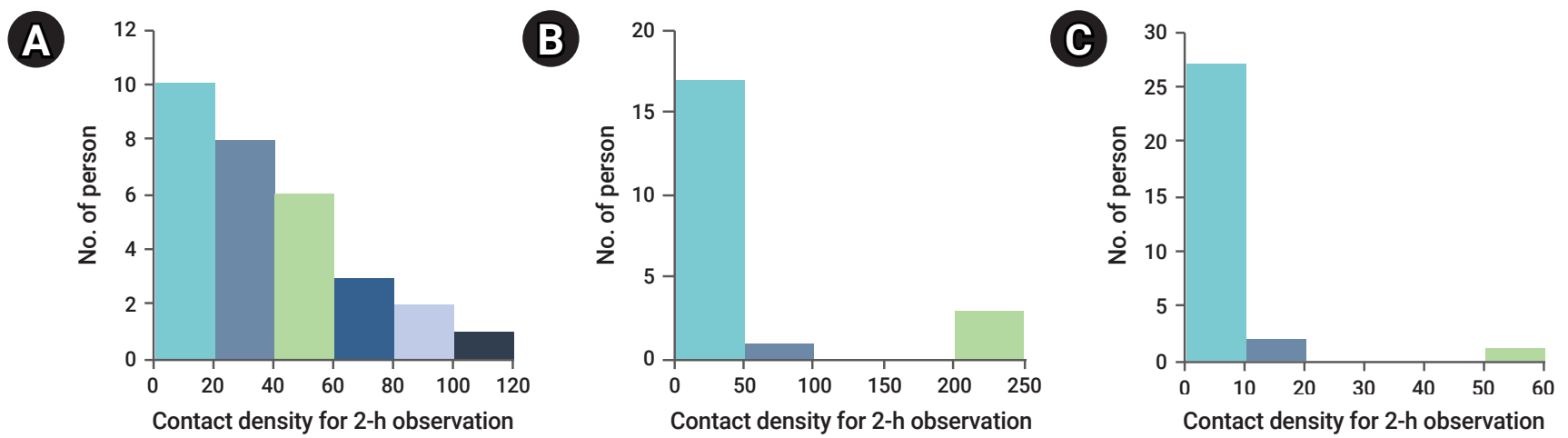

Figure. 1. Histogram for the distribution of contact density by subgroup items.

$\mathrm{Y}$ is the probability density of the frequency of a person. $\mathrm{X}$ is the contact density (frequency-duration/min) reflecting the contact frequency and contact duration during the 2-h observation time. (A) Category I, items used by the individual; (B) category II, items mainly used by the individual but occasionally used by other people; (C) category III, public use items.

Table 6. Differences in contact density of hand-to-environmental contact by participants' characteristics (2-h observation time, $n=4,532, N=30)$

\begin{tabular}{|c|c|c|c|c|}
\hline Variable & $N(\%)$ & \multicolumn{3}{|c|}{ Contact density (frequency-duration/min/person) } \\
\hline Sex & & 0.350 & $0.020^{*}$ & 0.966 \\
\hline Female & $19(63.3)$ & $29.5(17.4-55.9)$ & $36.4(24.4-58.4)$ & $0.5(0.1-1.4)$ \\
\hline Marital status & & $0.047^{\star}$ & 0.495 & 0.984 \\
\hline Age $(y)$ & & 0.065 & 0.229 & $0.035^{\star}$ \\
\hline $20-29$ & $13(43.3)$ & $46.1 \pm 28.6$ & $233.1(111.1-244.1)$ & $0.6(0.2-1.4)$ \\
\hline $30-59$ & $10(33.3)$ & $37.5 \pm 33.1$ & $22.0(11.5-37.0)$ & $0.1(0.0-0.3)$ \\
\hline$\geq 60$ & $7(23.3)$ & $14.4 \pm 11.9$ & $31.0(25.4-39.2)$ & $1.4(0.8-2.8)$ \\
\hline Job & & 0.854 & 0.488 & 0.093 \\
\hline Household monthly income (\#) & & $0.002^{\star \star}$ & 0.968 & 0.555 \\
\hline$<5,000,000$ & 15 & $13.5(6.0-28.5)$ & $29.1(18.8-43.1)$ & $0.6(0.3-1.4)$ \\
\hline$\geq 5,000,000$ & 13 & $52.6(43.9-72.4)$ & $35.8(19.6-43.2)$ & $0.2(0.1-1.5)$ \\
\hline Education & & 0.064 & 0.910 & $0.003^{\star \star}$ \\
\hline$\leq$ High school & 15 & $26.8 \pm 25.1$ & $31.9(22.7-42.8)$ & $1.4(0.6-4.5)$ \\
\hline$\geq$ College & 14 & $47.1 \pm 31.4$ & $30.1(15.2-132.7)$ & $0.2(0.0-0.7)$ \\
\hline
\end{tabular}

Data are presented as $p$-value, mean $\pm S D$, or median (interquartile range). If the normality test of the residuals showed normality, the mean $\pm S D$, and $t$-test or ANOVA were used, and if non-normality occurred, the median (interquartile range) and non-parametric Wilcoxon tests or the Kruskal-Wallis test were used.

SD, standard deviation; \#, Korean won.

${ }^{\text {a) }}$ Category I, items used by the individual (mobile phones, personal pens, glasses, etc.); category II, items mainly used by the individual but occasionally used by other people (papers, computer accessories, and utensils, etc.); category III, public use items (furniture surfaces, door handles, etc.).

${ }^{*} p<0.05,{ }^{*} p<0.01$.

In this study, HEC was shown to occur at a high frequency during indoor activities. Restriction of unnecessary environmental contacts may be strongly recommended, as previous studies have shown that reducing the contact rate is somewhat more effective than increasing ventilation for lowering infection risk [13,14].

Mobile phone contact (category I) accounted for the highest proportion of HEC, at $24.2 \%(n=1,143)$. Mobile phones can be 
a source of bacterial cross-contamination and a reservoir for infections [22]. Healthcare workers' use of mobile phones increases the risk of repetitive cyclic contamination between the hands and face (e.g., the nose, ears, and lips) [23]. Among Koreans, the average frequency per person of hand-to-facial contact during a 2-hour period was 46.5 (46.3\%) for mucosal contact (the eyes, mouth, and nose) and 53.8 (53.7\%) for nonmucosal contact [24]. The transmission of an infection after using a mobile phone is most likely when an individual touches a mucous membrane with his or her hands. In particular, mobile phones with keypads pose a higher risk of microbial contamination than touchscreen phones, as keypads harbor pathogenic bacteria [23]. Studies have revealed that between $9 \%$ and $25 \%$ of mobile phones used by healthcare workers are contaminated with microorganisms [25]. Mobile phones are an indispensable means of communication in our daily lives; they also provide us with internet access (e.g., for social media updates) and are a tool for taking photos and videos. Therefore, mobile phones should be regularly and thoroughly cleaned or disinfected. Moreover, individuals should avoid habitually touching the mucous membranes of their mouth, nose, and eyes, and should wash their hands thoroughly after touching their mobile phones.

Considering the high risk of cross-contamination among category II and II items, furniture surface contact and paper contact occurred at high frequencies, accounting for $14.7 \%$ ( $n=696)$ and $12.7 \%(n=603)$ of total environmental contact, respectively. The average contact frequency and contact duration of furniture surfaces (category $\Pi$ ) were rather high, compared with mobile phones in category I. It can therefore be said that the most cross-contamination among category II surfaces occurred with furniture surfaces, despite the fact that there were fewer environmental items in category II than in category I or II. Regular surface cleaning has been shown to reduce the influenza A infection rate by $2.14 \%$, which is more effective than handwashing [17]. Pieces of furniture, such as desks and chairs in classrooms or churches, are common-use items and can be a route for the spread of infections. Therefore, stricter and more regular thorough cleaning and hygiene practices must be followed to prevent infection transmission through furniture surfaces in public spaces. Unlike other studies $[10,12,26]$, a low contact frequency with handles or doorknobs was demonstrated in this study; this can be attributed to conducting the observations in a sedentary environment within a limited timeframe in an indoor space where outward movement was restricted.

Globally, money is one of the items most frequently passed from hand to hand. During this exchange, money can become contaminated and may thus play a role in the transmission of microorganisms between people [27].
Therefore, people should be advised to thoroughly wash their hands after touching money. However, the frequency of hand contact with money was not observed in this study, because the study was conducted in an indoor environment-for example, offices, classrooms, churches-and did not include banks or marketplaces.

The average contact duration at each contact (seconds per contact per person) was high for category I, while contact frequency, total contact duration (seconds per person), and contact density within the 2-hour period were highest for category II. This means that the risk of transmitting the infection by HEC to the person himself or herself was shown to be most likely via category I items, while the risk of transmission to other people was most likely via category II items. Therefore, regular and strict cleansing or disinfection of category I items, such as mobile phones, may be required. Moreover, sharing personal items with other people should be avoided when possible during indoor activities.

The contact density (frequency-duration/min) of HEC according to the general characteristics of the participants in this study can guide differentiated infection control strategies. For example, singles and individuals with a household income of more than 5 million Korean won had a high contact density with items from category I (e.g., mobile phones); women had a higher contact density for category II items; and seniors aged 60 years and older and people with less than a high school education were likely to have a high degree of contact with items in category III (e.g., furniture surfaces). These findings suggest that sex, age, education level, and economic status may influence the risk of HEC transmission to others. These results can be used for public awareness and education campaigns. However, further studies are necessary to confirm the abovementioned conclusions, given the limited number of participants in this study.

The histogram confirming the contact density distributions shows that categories II and III had exponential distributions (Figure 1). This means that most people were distributed at low contact densities, but it is important to note that a few people had high contact density. In particular, the items in categories II and III, although small in number, are likely to be super-propagators during an epidemic of an infectious disease; therefore, they need to be identified and specially managed.

This study has certain limitations that should be considered. First, the video recording was conducted for only 2 hours, with a limited number of persons (30 participants) in indoor settings such as classrooms, offices, and churches. Therefore, this study did not measure every type of contact during all types of indoor activities among all Koreans. Second, 
there is a limitation to generalizing the characteristics of the participants with high contact density, as only 30 participants were evaluated. Thirty is a reasonable sample size for video observation studies, as typically only 10 to 20 participants are enrolled for these kinds of studies due to practical challenges in carrying out the $[18,28]$. Further studies will be conducted to determine HEC patterns in various everyday indoor activities in different situations with a larger number of participants.

Despite these limitations, this study contributes in significant ways. First, characteristics of HEC in indoor activities were evaluated, and the data pointed to significant variables for preventing the spread of infection via HEC, according to environmental categories. These significant variables may be useful in developing public education programs or HEC policies to prevent the spread of infections. Moreover, the classification criteria for environmental contact items and contact indicators representing contact strength will be useful for further HEC studies. This classification criterion of items in the environment and contact indicators such as contact density introduced in this study can be useful in conducting similar studies. Significantly, the world is currently in the middle of the coronavirus disease 2019 pandemic [29]; the results of this study on HEC are therefore highly relevant and can be very useful for preventing the spread of infections via HEC.

\section{Conclusion}

This study determined that HEC frequently occurred and offered various useful insights regarding HEC and the spread of infections during indoor activities. These findings will be useful in developing precautions to prevent the spread of infections via HEC. The most frequent HEC items, in descending order, were found to be category I (e.g., mobile phones), category $\amalg$ (e.g., furniture surfaces), and category II (e.g., paper and computer accessories). Contact density was highest in category II. Regular and strict cleansing of mobile phones and/or a disinfection strategy for mobile phones is recommended. It is also important to avoid sharing personal items with others. Personal characteristics such as household income, marital status, sex, age, and level of education may be taken into account when educating the public regarding the precautions that would limit HEC. In particular, items in categories II and III, although few in number, are likely to be super-propagators during an epidemic of an infectious disease; therefore, they need to be identified and specially managed. These results can serve as strong evidence for the need to regularly cleanse or disinfect environmental items, restrict unnecessary HEC, and maintain strict hand hygiene.

\section{Notes}

\section{Ethics Approval}

This study was approved by the IRB from the Sunchon National University (IRB No: 1040173-201712-HR-033-02). Informed written consent was obtained from all individual participants before and after video recording. All video analyses were secured to protect the identities of the participants after video reading. Participants gave their written informed consent to participate in the study. Participation in the study was voluntary, and consent could be withdrawn at any time without giving reasons or suffering disadvantages.

\section{Conflicts of Interest}

The authors have no conflicts of interest to declare.

\section{Funding}

This research was supported by a fund (code\# 2017P140300) from the Korea Centers for Disease Control and Prevention (KCDC).

\section{Availability of Data}

The datasets are not publicly available but are available from the corresponding author upon reasonable request.

\section{References}

1. Boyce JM, Pittet D, Healthcare Infection Control Practices Advisory Committee, et al. Guideline for hand hygiene in health-care settings: recommendations of the Healthcare Infection Control Practices Advisory Committee and the HIPAC/SHEA/APIC/IDSA Hand Hygiene Task Force. Am J Infect Control 2002;30:S1-46.

2. World Health Organization (WHO). WHO guidelines on hand hygiene in health care: first global patient safety challenge clean care is safer care. Geneva: WHO; 2009.

3. Phan LT, Maita D, Mortiz DC, et al. Environmental contact and selfcontact patterns of healthcare workers: implications for infection prevention and control. Clin Infect Dis 2019;69(Suppl 3):S178-84.

4. Suleyman G, Alangaden G, Bardossy AC. The role of environmental contamination in the transmission of nosocomial pathogens and healthcare-associated infections. Curr Infect Dis Rep 2018;20:12.

5. Ren SY, Wang WB, Hao YG, et al. Stability and infectivity of coronaviruses in inanimate environments. World J Clin Cases 2020; 8:1391-9.

6. Chowdhury D, Tahir S, Legge M, et al. Transfer of dry surface biofilm in the healthcare environment: the role of healthcare workers' hands as vehicles. J Hosp Infect 2018;100:e85-90.

7. Weber DJ, Anderson D, Rutala WA. The role of the surface environment in healthcare-associated infections. Curr Opin Infect Dis 2013;26:33844.

8. Weber DJ, Rutala WA, Miller MB, et al. Role of hospital surfaces in the transmission of emerging health care-associated pathogens: norovirus, Clostridium difficile, and Acinetobacter species. Am J Infect Control 2010;38(5 Suppl 1):S25-33.

9. La Rosa G, Fratini M, Della Libera S, et al. Viral infections acquired 
indoors through airborne, droplet or contact transmission. Ann Ist Super Sanita 2013;49:124-32.

10. Winther B, McCue K, Ashe K, et al. Environmental contamination with rhinovirus and transfer to fingers of healthy individuals by daily life activity. J Med Virol 2007;79:1606-10.

11. Cho SY, Kang JM, Ha YE, et al. MERS-CoV outbreak following a single patient exposure in an emergency room in South Korea: an epidemiological outbreak study. Lancet 2016;388:994-1001.

12. Rheinbaben F, Schünemann S, Gross T, et al. Transmission of viruses via contact in a household setting: experiments using bacteriophage straight phiX174 as a model virus. J Hosp Infect 2000;46:61-6.

13. Adams CE, Smith J, Watson V, et al. Examining the association between surface bioburden and frequently touched sites in intensive care. J Hosp Infect 2017;95:76-80.

14. Sze-To GN, Yang Y, Kwan JK, et al. Effects of surface material, ventilation, and human behavior on indirect contact transmission risk of respiratory infection. Risk Anal 2014;34:818-30.

15. Kurgat EK, Sexton JD, Garavito F, et al. Impact of a hygiene intervention on virus spread in an office building. Int J Hyg Environ Health 2019; 222:479-85.

16. Lei H, Xiao S, Cowling BJ, et al. Hand hygiene and surface cleaning should be paired for prevention of fomite transmission. Indoor Air 2020;30:49-59.

17. Zhang N, Li Y. Transmission of influenza A in a student office based on realistic person-to-person contact and surface touch behaviour. Int J Environ Res Public Health 2018;15:1699.

18. Ferguson AC, Canales RA, Beamer P, et al. Video methods in the quantification of children's exposures. J Expo Sci Environ Epidemiol 2006;16:287-98.

19. R Core Team. R: a language and environment for statistical computing [Internet]. Vienna: The R Foundation for Statistical Computing; 2017 [cited 2019 Feb 14]. Available from: http://www.r-project.org.
20. Warnes GR, Bolker B, Bonebakker L, et al. gplots: various R programming tools for plotting data. R Packag Version 2009;2:1.

21. Korean Statistical Information Service. Report on the time use survey volume [Internet]. Daejeon: Statistics Korea; 2019 [cited 2021 Mar 30]. Available from: https://kosis.kr/publication/publicationThema. do?pubcode $=$ LS.

22. Pal P, Roy A, Moore G, et al. Keypad mobile phones are associated with a significant increased risk of microbial contamination compared to touch screen phones. J Infect Prev 2013;14:65-8.

23. Ulger F, Dilek A, Esen S, et al. Are healthcare workers' mobile phones a potential source of nosocomial infections? Review of the literature. J Infect Dev Ctries 2015;9:1046-53.

24. Oh HS, Ryu M, Yang Y, et al. Analysis of transmission patterns of infection according to contact behavior in daily life. Cheongju: Korea Centers for Disease Control and Prevention; 2018. Report No.: 111352159-001049-01.

25. Brady RR, Verran J, Damani NN, et al. Review of mobile communication devices as potential reservoirs of nosocomial pathogens. J Hosp Infect 2009;71:295-300.

26. Barker J, Vipond IB, Bloomfield SF. Effects of cleaning and disinfection in reducing the spread of Norovirus contamination via environmental surfaces. J Hosp Infect 2004;58:42-9.

27. Gedik H, Voss TA, Voss A. Money and transmission of bacteria. Antimicrob Resist Infect Control 2013;2:22.

28. Kwok YL, Gralton J, McLaws ML. Face touching: a frequent habit that has implications for hand hygiene. Am J Infect Control 2015;43:112-4.

29. World Health Organization (WHO). Coronavirus disease (COVID-19) advice for the public [Internet]. Geneva: WHO; 2020 [cited $2020 \mathrm{Apr}$ 29]. Available from: https://www.who.int/emergencies/diseases/novelcoronavirus-2019/advice-for-public. 